\title{
La investigación formativa y la didáctica para la enseñanza y el aprendizaje del mercadeo
}

\author{
Formative Research \\ And Didactics for the Teaching and Learning of Marketing \\ A pesquisa formativa \\ e a didática para o ensino e a aprendizagem do marketing
}

Recibido marzo 6 de 2018. Aprobado mayo 11 de 2018

Cristian Hernández Gil*

http://orcid.org/0000-0001-6512-2453

Colombia

Eduardo Polanía González**

http://orcid.org/0000-0001-5361-2578

Colombia

- Para citar este artículo:

Hernández-Gil, Cristian y

Polanía-González, Eduardo. (junio, 2019). La investigación formativa y la didáctica para la enseñanza y el aprendizaje del mercadeo. Ánfora, 26(46), 39-64. DOI: https://doi.org/10.30854/ anf.v26.n46.2019.552 Universidad Autónoma de Manizales. ISSN 0121-6538/ e-ISSN 2248-6941

\section{Resumen}

Objetivos: identificar y describir la importancia de la investigación formativa y el uso de la didáctica para fortalecer el proceso de enseñanza-aprendizaje del mercadeo como asignatura en la educación superior. Metodología: se realizó un estudio descriptivo de enfoque cualitativo a través de una revisión documental y el desarrollo de dos filtros de información con base en dos criterios establecidos por los autores de esta investigación: relevancia del texto sobre las categorías de análisis y proporcionalidad en la distribución de los textos en dichas categorías. Resultados: el mercadeo

\footnotetext{
** Administrador de Empresas. Profesor Universidad de la Amazonia. Florencia, Caquetá. Maestrando en Ciencias de la Educación.Correo: cris.hernandez@udla.edu.co, cristianhgil1@gmail.com

** Administrador de Empresas de la Universidad de la Amazonia. Correo: eduarfil@hotmail.com
} 
actualmente se enseña de diferentes maneras, pero se resaltan dos primordialmente: a) las que posicionan al docente como actor activo del proceso formativo y, al estudiante como actor pasivo, quien solamente se dedica a recibir información; y b) el enfoque hacia la equidad entre la enseñanza y el aprendizaje para que tanto el maestro como el estudiante se conviertan en elementos activos del proceso. Conclusiones: el mercadeo se debe enseñar-aprender usando tecnologías de la información y la investigación formativa y tratando de simular situaciones reales provistas por el sector empresarial.

Palabra claves: Marketing; Enseñanza de Mercadeo; Investigación; Didáctica.

\section{Abstract}

Objective: to identify and describe the importance of formative research and didactics in strengthen the teaching-learning process of marketing as a subject in higher education. Methodology: a qualitative descriptive study was carried out. Data collection was done through a documentary review and the development of two information filters based on two criteria: text relevance based on the categories of analysis and the proportionality in the text distribution of such categories. Results: marketing is currently taught in different ways, but there are primarily two ways: a) those placing the professor as an active party in the teaching process, and the student as a passive party who only receives information; and b) the approach to equity between teaching and learning, thus both the professor and the student become active parties in the process. Conclusions: marketing should be taught-learned by using information technologies and formative research in collaboration with an intention to simulate real situations provided by the current business sector.

Keywords: Marketing; Marketing Teaching; Research; Didactics.

\section{Resumo}

Objetivo: identificar e descrever a importância da pesquisa formativa e o uso da didática para fortalecer o processo de ensino-aprendizagem do marketing como disciplina no ensino superior. Metodologia: foi realizado um estudo descritivo de abordagem qualitativa, por meio de uma revisão documental e do desenvolvimento de dois filtros de informação, a partir de dois critérios estabelecidos pelos autores desta pesquisa: relevância do texto nas categorias de análise e proporcionalidade na distribuição dos textos dessas categorias. Resultados: o marketing é ensinado atualmente de maneiras diferentes, mas duas são destacadas: a) aquelas que 
posicionam o professor como ator ativo no processo de formação e ao estudante como ator passivo, que apenas recebe informações; e b) a abordagem que procura a equidade entre ensino e aprendizagem, de modo que tanto o professor quanto o estudante se tornem elementos ativos do processo. Conclusões: o marketing deve ser ensinado -aprendido usando tecnologias da informação e pesquisa formativa, e tentando simular situações reais fornecidas pelo atual setor empresarial.

Palavras-chave: Marketing; Ensino de Marketing; Pesquisa; Didática. 


\section{Introducción}

Zuleta (2010) asegura que "Un hombre que pueda pensar por sí mismo, apasionarse por la búsqueda del sentido o por la investigación, es un hombre mucho menos manipulable" (p. 10). Por eso, hablar de investigación es cada vez más importante, más aún, porque supone la adopción de una competencia técnica y profesional. La investigación, entonces, debe ser considerada en todas las etapas y contextos de aprendizaje del ser humano para ser usada como una herramienta que ayuda a solucionar los problemas, cotidianos y complejos, mediante hallazgos que generen desarrollo o mejoramiento de la calidad de vida.

El objetivo de este artículo es describir cómo se pueden establecer metodologías más adecuadas para enseñar y aprender mercadeo o marketing (por su expresión en inglés), desde la investigación formativa y la didáctica, con miras a la adquisición del conocimiento y a la apropiación del mismo para su debida aplicación en el medio externo, lugar de desarrollo del futuro profesional. Así, la pregunta en torno a la cual gira esta investigación es ¿Cuál es la importancia de la aplicabilidad de la investigación formativa y la didáctica para la enseñanza efectiva del mercadeo y su debido y adecuado aprendizaje?

Actualmente, las universidades no solamente tienen la misión de impartir conocimiento. Según Sandoval (2010), entre las funciones que distinguen a estas de las demás instituciones de educación superior, está la investigación, que permite vincular nuevos conocimientos al ejercicio de la docencia. Esto implica atribuir a la investigación formativa la importancia debida, para permitir nuevas formas de resolver problemas en diferentes contextos, sobre todo, en lo social, económico y empresarial. En lo relativo a la formación de profesionales, se espera que estos tengan una educación ligada al campo laboral, relacionada con el lugar donde cada uno desarrollará su actividad, luego de convertirse en gestores del cambio en cada ciudad, región o, incluso, en cada país.

A propósito de la investigación formativa, el Consejo Superior Universitario de la Universidad de Antioquia (1994) afirma que la docencia, fundamentada en la investigación, permite formar a los estudiantes en los campos disciplinarios y profesionales de su elección, mediante el desarrollo de programas curriculares y el uso de métodos pedagógicos que facilitan el logro de los fines éticos y académicos de la universidad. Lo anterior, determina el carácter social de los claustros de educación superior, en función de las responsabilidades científicas y morales de los profesores frente a sus estudiantes, a la institución y a la sociedad. En este caso, se plantea la aplicación de la investigación en la enseñanza del mercadeo, en contraste con el ejercicio académico que, según Camacho (2015), en las universidades públicas está basado en las clases magistrales y en el dominio de conocimientos del que asume el rol de enseñar. 
De acuerdo con lo anterior, se hace una revisión documental de tres categorías de análisis, a saber: la importancia de la educación en la investigación, la didáctica y el mercadeo y las tendencias en el perfil de profesionales en mercadeo. Con este documento se logra nuevos enfoques para personas e instituciones que forman en mercadeo, con el fin de que asuman la función de concederle su debida participación al estudiante con los elementos de corte investigativo y didáctico.

En las aulas, el mercadeo se enseña con diferentes connotaciones y orientaciones que son importantes analizar para entender cuál sería la mejor forma de hacerlo para el cumplimiento de dos objetivos: desarrollar un aprendizaje efectivo que promueva el perfeccionamiento de las habilidades profesionales en mercadeo y, por otro lado, incluir la investigación formativa para la creación de nuevos conocimientos relacionados con las tendencias del mercado (espacio donde los consumidores demandan y las empresas ofertan).

Es importante mencionar, que los resultados presentados a continuación son parte del análisis preliminar de una investigación aplicada realizada en el programa de Administración de Empresas de la Universidad de la Amazonía denominada "Propuesta didáctica para la articulación de la investigación formativa y la enseñanza del mercadeo”.

\section{Metodología}

El desarrollo de esta investigación se fundamenta en un estudio de tipo descriptivo en el que se identificaron y perfilaron las características propias de la investigación formativa y la didáctica para ser usadas como métodos de enseñanza en el área de mercadeo. Se partió de una revisión documental, con un enfoque cualitativo estableciéndose tres fases para la solución del problema de investigación planteado:

La primera fase consistió en la identificación de tres categorías de análisis, determinadas por los autores así: primero, se reconocieron los temas centrales a analizar y, sobre ellos, se establecieron relaciones de tipo causal; es decir, que entre las categorías se puede examinar otros subtemas que engloban de manera holística esta investigación. Al final de esta etapa, se seleccionaron tres categorías: la importancia de la educación en la investigación, la didáctica y el mercadeo y las tendencias en el perfil de profesionales en mercadeo. 
La segunda fase consistió en la recolección de una base de datos con cincuenta (50) referencias bibliográficas agrupadas en cada categoría de análisis. Así, se construyó una tabla en Microsoft Excel con todos los elementos de cada referencia como el autor, el año de publicación, el resumen, el objetivo central, la cita textual, el tipo de cita, el tipo de documento, la bibliografía o forma de citar, entre otros.

En la tercera fase, se agruparon las referencias en cada categoría, buscando cierto grado de proporcionalidad (cantidad) para cada una. Y el resultado se evidencia en la siguiente tabla:

Tabla 1. Proporción de citas o referencias según la categoría de análisis

\begin{tabular}{l|c}
\multicolumn{1}{c|}{ Categoría de Análisis } & Numero de referencias \\
\hline $\begin{array}{l}\text { La importancia de la educación en la } \\
\text { investigación }\end{array}$ & 18 \\
\hline La didáctica y el mercadeo & 23 \\
\hline $\begin{array}{l}\text { Las tendencias en el perfil de profesionales } \\
\text { en mercadeo }\end{array}$ & 9
\end{tabular}

Fuente: elaboración propia.

En esta última fase, los autores a través del uso del software Atlas.ti realizaron una red semántica para establecer las relaciones entre las tres categorías y el fin último de este estudio, la cual se evidenciará en el apartado de resultados.

\section{Resultados}

\section{La importancia de la investigación en la educación}

Zuleta (2010) asevera que "El niño es un investigador según la definición de Freud. Pero si el maestro tiene la respuesta de antemano, el alumno pierde las condiciones para investigar porque lo que tiene que hacer es buscar la respuesta que exige el maestro para adecuarse a ella” (p. 20). Esta crítica pone al docente en el papel de intimidador, que busca que el estudiante haga en función de lo que él le exige, y esto es muy distinto a una verdadera relación con el saber.

Desde este punto de partida, es importante considerar la investigación como un fenómeno indispensable para la formación en el aula de clases. Por ejemplo, Jessup y de Castellanos (2017) aseguran que la investigación no se centra en el desarrollo de conocimientos en una disciplina particular, sino en una prác- 
tica sistemática asumida como resultado de la interrelación permanente entre la reflexión y la acción crítica. Con esto se entiende que la investigación es un elemento importante en la educación, pues crea cimientos en los procesos de formación integral de los que se están educando, teniendo en cuenta la realidad que determina sus condiciones de vida. Esto aproxima a la verdadera razón por la que se debe incluir la investigación formativa en las aulas de instituciones de educación superior: cuando se investiga se desarrollan habilidades para pensar y actuar críticamente, con sentido ético, afectividad y sensibilidad, tanto en lo individual como en lo social.

De esta manera, la investigación formativa es parte crucial del proceso de enseñanza y aprendizaje en cualquier situación educativa. Para Moncada y Pinilla (2006) el docente debe tener la investigación como un pilar de su quehacer y, por tanto, convertirla en una herramienta didáctica para el aprendizaje de las diferentes disciplinas y profesiones. En este sentido, la investigación hace parte de una de las funciones centrales del docente, que no solo se debe quedar en el ejercicio individual, sino que es menester integrarla mediante la interacción con sus estudiantes y como metodología de su quehacer docente. En ambos casos, la investigación permite crear espacios de nuevos conocimientos.

Así mismo, Giraldo, Abad, y Díaz (2004) explican cómo la investigación no es un proceso que debe ser realizado, por el docente, por obligación o para cumplir una función más como integrante de un claustro universitario. Sino que ésta, según los autores, debe hacerse con calidad. Esta característica abarca todas sus funciones y actividades principales como: calidad de enseñanza, de formación e investigación, calidad de su personal docente y de los programas, y calidad de aprendizaje como corolario de la enseñanza.

La calidad también involucra la responsabilidad del docente investigador de presentar los resultados de sus estudios y fomentar una "cultura de visibilidad en la producción académica”. Sobre esto, la División de Investigación de la Universidad Nacional de Colombia Sede Bogotá (1999) establece que

El docente investigador y su equipo, deben comprender la importancia de la socialización de los resultados de sus investigaciones, que van desde los encuentros internos de departamento y facultad, pasando por congresos de diversos niveles, hasta la publicación en revistas especializadas, que finalmente deben ser referenciadas por otros autores nacionales e internacionales (p. 25).

Por consiguiente, una investigación hecha por el docente y que vincule la participación de los estudiantes, promueve la calidad en los procesos educativos originando lo que Granés (2001) llama el fomento de una cultura de la investigación, la cual promueve en profesores y estudiantes un espíritu de crítica frente 
al conocimiento. Esto implica, además, análisis detenido de las teorías o paradigmas que están validados en un momento dado por las comunidades científicas y el planteamiento de inquietudes que probablemente no estén resueltas.

Clark (1998) ya había resaltado la importancia de la investigación en los institutos universitarios. Según él, la mayor parte de las actividades de enseñanza y aprendizaje, en estos niveles de formación, se llevan a cabo básicamente mediante un involucramiento directo de la investigación y una capacitación preparatoria estrechamente relacionada con los proyectos de disertación de nuevos conocimientos vigentes, por ejemplo, en laboratorios o seminarios, tesis estudiantiles y cursos que siguen métodos investigativos.

Neumann (1992), por su parte, reconoce el efecto positivo de la inclusión de la investigación en la docencia. Todo ello consolida la idea de que ambas funciones son inseparables en el aula, lo que crea dos tipos de conexiones: primero, hay una tangible que implica la transmisión de conocimientos avanzados y habilidades de investigación a los estudiantes. La segunda conexión es intangible y se relaciona con el desarrollo de actitudes y enfoques positivos hacia la investigación y el aprendizaje.

Pina (2002) complementa lo anterior al establecer la relación directa entre la docencia y la investigación a través de dos vías: cuando el profesor aporta ejemplos y resultados de sus investigaciones en clase o cuando en el proceso de enseñanza recibe algún insight que pueda trasladar a su línea de investigación. Pina también considera la existencia de otra relación indirecta que se establece cuando un profesor con experiencia investigadora disemina o promueve actitudes críticas y entusiasmo hacia el conocimiento que está impartiendo dentro de su materia.

Ahora bien, hasta el momento se ha formulado cómo la investigación asume su papel dentro de la educación, en especial en el nivel superior. Pero ¿qué pasa con la opinión de los estudiantes frente al tema? Lindsay, Breen y Jenkins (2002) muestran que todos los estudiantes universitarios que participaron en su estudio opinaron que la investigación produce más beneficio que perjuicios; así mismo, todos expresaron que la actividad investigadora promueve la difusión del conocimiento, la credibilidad, el entusiasmo y la motivación. Es de esperarse, entonces, que los estudiantes elogien el interés, relevancia y utilidad de la investigación en los contenidos de su aprendizaje y terminen por reconocer los efectos negativos que tiene sobre la enseñanza la falta de disponibilidad de algunos profesores para el apoyo en investigación.

Así pues, la investigación se ha vuelto indispensable, incluso para el desarrollo de las naciones. Moreno y Ruiz (2010) consideran que el crecimiento económico en América Latina requiere de inversión y aplicación del progreso 
científico y tecnológico para modernizar los procesos productivos de la región. Con ello, establecen tres elementos claves para su debida aplicación:

1) la infraestructura científica; 2) la oferta de personal de investigación altamente calificado, y 3) una estrecha y funcional vinculación entre los centros de investigación y las empresas productivas. En otros términos, la competitividad de una región o nación parte del interés público por mejorar los capitales locales -físico y humano- que puedan llevar a cabo la investigación y el desarrollo, así como crear las condiciones para asegurarse de que los centros de ciencia y tecnología tengan vínculos relevantes, efectivos y eficientes con el sector empresarial local.

En otro sentido, García (2000) apoya la idea de que la investigación aporte a la construcción colectiva del conocimiento a través de los procesos de "innovación y selección crítica", ya que esto hace que la creación conceptual y el discernimiento reflexivo de los alumnos se incrementen extraordinariamente. Esta idea es complementada por Carvajal (2010) al afirmar que "la investigación abre nuevos horizontes al alumno, completa la información ofrecida por los docentes y permite una especialización personal enriquecedora" (p. 21). En esta función constructiva, Agazzi (2001) propone una investigación interdisciplinar que parta de la individualización del problema y de los aspectos del mismo que requieren del enfoque de cada disciplina formulada; esto permite una relación con otros saberes y, por ende, que la educación sea integral.

Como indica Martínez (2004), uno de los signos de la época actual es la multiplicidad de posturas científicas en cuanto a los saberes, filosofías, enfoques, disciplinas, especialidades, teorías, métodos y técnicas de investigación. Esto lleva a la necesidad de repensar la coordinación en la formación profesional, para unificar criterios y establecer una profunda unión e integración con base en el diálogo; que ponga en común "verdades" distintas sobre la realidad que se investiga y que permita descubrir significados, a partir de la discusión dentro de comunidades científicas.

López (2015), además, reconoce que la formación investigativa del futuro docente se asume como el proceso que permite a éste adquirir las competencias necesarias para aprehender la situación educativa; igualmente, a buscar soluciones según la problematización de la práctica socioeducativa con base en la investigación dirigida a la protección del medio ambiente, la biodiversidad y el entorno sociocultural. Así mismo, Pérez (2003) establece que, en las economías latinoamericanas, especialmente la venezolana, es importante replantear la formación investigativa del futuro docente, con la posibilidad de crear nuevos espacios para la discusión de la cultura experiencial, las vivencias y las cotidianidades del proceso de enseñanza y aprendizaje que representan una creación permanente hacia la investigación como base de la enseñanza. 
Según Sánchez (2007), la sociedad reclama de la ciencia, la tecnología y la innovación, una investigación orientada a la solución de problemas y aportes verificables en respuesta a sus necesidades. Para Colombia, un país con grandes desafíos sociales, económicos y ambientales como hambre, desnutrición, pobreza extrema, desempleo, violencia y deterioro ambiental, entre otros, esto se constituye en un reto para la comunidad científica y tecnológica.

\section{La didáctica y el mercadeo}

En este apartado se inicia con una distinción del término didáctica para luego ingresar a la descripción de métodos utilizados actualmente para el desarrollo progresivo de la enseñanza del mercadeo.

Civarolo (2008) expresa la definición de la didáctica en función de un aspecto teleológico de la educación y la conceptualiza como el

Arte que se realiza en el dominio de la praxis. Un arte que debe ser comprendido como un hacer, más que un producir, un hacer en que la educación tiende a promover la personalización del educando, buscando que se desarrolle ese centro interior que posee para que sea cada vez más él mismo y decida mejor de sí (p.148).

Rosselló (2005) explica cómo la didáctica general ha evolucionado a partir de la década de los ochenta cuando sufre un gran cambio de perspectiva en el que convergen diferentes hechos, como son: a) En el contexto científico y epistemológico, surgen nuevas formas de entender y construir el conocimiento científico. b) En el contexto social, la incursión en la postmodernidad y la sociedad del conocimiento, propicia la emergencia de nuevas maneras de pensar la realidad c) En el contexto profesional, se produce un cambio de roles en la función docente y en su contexto de trabajo (condiciones organizativas de la escuela, emergencia de nuevos ámbitos formativos).

Todo lo anterior, cobra sentido en la medida en que la didáctica toma relevancia en el proceso de aprendizaje, el cual une de manera holística dos sujetos: el docente y los estudiantes; por tanto, la didáctica es la herramienta dialógica con la que el maestro desempeña su labor para dar participación más activa a los educandos.

De esta forma, Moreno (2011) le da importancia a la didáctica en la enseñanza superior. La educación universitaria tiene su propia concreción; por eso, requiere una didáctica distintiva que posibilite el aprendizaje de los alumnos, en su mayoría adultos, con conocimientos y experiencias previas, motivaciones y expectativas diversas respecto a su proyecto personal y profesional. Todo lo 
anterior, implica la concepción de una didáctica que no solamente se quede en el componente metodológico, sino que además logre evidenciar la utilidad, para el estudiante y su proyecto de vida, sobre todos los saberes que le son inculcados o transmitidos. Esto admite incluir, un nuevo concepto, el ámbito andragógico del aprendizaje significativo.

Ahora, el estudiante, en sus estudios superiores, siempre trae consigo una experiencia previa, sobre todo si ya ha pasado de cierta edad. Este aprendizaje vela por el desarrollo de unas necesidades que desea cubrir. Para Sánchez (2017)

El modelo andragógico potencializa el aprendizaje del adulto, en el que el conocimiento, así como, la utilización de las metodologías didácticas convenientes, acompañada del empleo de técnicas y procedimientos para el aprendizaje fomenten en estos estudiantes el aprovechamiento del tiempo, poniendo a su disposición un proceso orientado por sus intereses, necesidades y expectativas, donde el docentefacilitador sea un guía u apoyo del grupo (p. 1).

Esto implica hablar de la práctica y la reflexión, como atributos que generan responsabilidad, al estudiante, de conocer y analizar la realidad en la que vive orientando sus procesos de aprendizaje, es decir, forjando su madurez como individuo.

Así mismo, Donoso y Vaca (2017) valiéndose de la ilustración andragógica, afirman que el adulto que enseña al adulto entiende que puede aplicar la enseñanza como un facilitador ante un alumno capaz de generar reflexiones propias. El profesor sería así además un promotor de desarrollo y cambio ante sus estudiantes, concediéndoles la libertad suficiente para que aprendan entre ellos de sus propias experiencias significativas. Es decir, no se trata de imponer que aprendan o que investiguen, puesto que se trata de un asunto de deseo y de necesidad, sino más bien que el estudiante lo asuma como algo propio y lo realice a cabalidad.

Aun así, "en la andragogía, el adulto decide qué aprender y es él mismo quien traza los objetivos y metas a alcanzar" (Villadiego et al., 2017, p. 318). Esto constituye otro principio más de la enseñanza del mercadeo, utilizando una didáctica en la que el mismo adulto construye saberes para orientarse en situaciones de incertidumbre, asumiendo el rol de profesional en marketing, desplegando su creatividad moviéndose sobre su cotidianidad, pues antes que satisfacer necesidades, inicialmente se establece como un consumidor, en un mercado cada vez más complejo y competitivo. 
En otro aspecto, Ortegón (2013) describe algunas situaciones que persisten actualmente en la enseñanza del mercadeo, en claustros latinoamericanos (ver figura 1):

1.Inicialmente, el docente no es especialista en el tema o no está formado en un pregrado con énfasis en mercadeo, por ende, aborda el tema desde otro saber y esto hace que el conocimiento se trabaje desde un contexto general.

2.Se trabaja la temática desde el punto de vista de las variables del marketing mix que plantean los principales autores del tema como Kotler y Stanton (producto, precio, plaza y publicidad o promoción), pero se aleja de conceptos prácticos y de las tendencias de la era actual, como por ejemplo, el mercadeo internacional, neuromarketing, marketing relacional, marketing político, marketing social, marketing ambiental, marketing industrial, marketing multinivel, mercadeo electrónico, marketing participativo, entre otros.

3.El uso de estudios de casos de contextos internacionales (marcas desconocidas) ajenos a la realidad de mercados nacionales inclusive locales.

4.Los textos con conocimientos más importantes de la materia como Fundamentos de marketing (Kotler, 2003), La guerra de la mercadotecnia (Ries y Trout, 1999), entre otros, generalmente tratan casos y situaciones empresariales de décadas de los ochentas o noventas, los cuales han perdido vigencia, pues desconocen conceptos como globalización, interconexión, ciberespacio y cibercultura, que influyen en el desarrollo del mercado y en su conocimiento.

5.Los docentes universitarios, permanentemente, desarrollan su trabajo con metodologías didácticas tradicionales, carentes de aprendizaje significativo y utilidad real por parte de los alumnos.

6.Las personas han cambiado el uso de los medios de comunicación, de forma que los medios convencionales han perdido representatividad en el consumo, el cual ha pasado al uso de herramientas más avanzadas y rápidas, segmentadas por temas y con mayor grado de interés para el espectador (p. 5). 
Figura 1. Elementos del sistema que persisten de la enseñanza del mercadeo

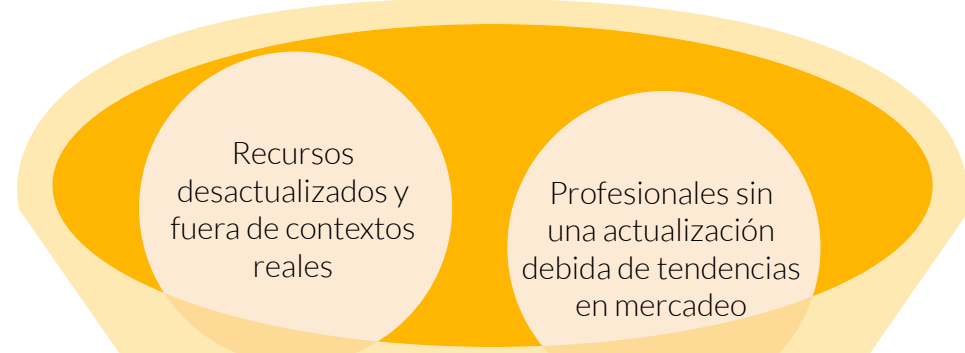

Nuevas formas

de hacer marketing

poco analizadas en

las aulas

\section{Fenómeno persistente en la} enseñanza del mercadeo

Fuente: elaboración propia con base en Ortegón (2013)

La didáctica en la enseñanza del mercadeo, entonces, debe promover la comprensión de las concepciones teóricas que se relacionan, por ejemplo, con el análisis del comportamiento del consumidor (satisfacer necesidades), a través de expresiones prácticas y habilidades investigativas que acerquen al educando a una sensibilización y al entendimiento de su entorno. De lo anterior, se evidencian algunas prácticas de corte didáctico para la enseñanza del marketing.

Camacho (2015), por su parte, resalta la importancia del uso del estudio de casos. Frente a esto, postula una la alternativa didáctica que hace del proceso pedagógico un fenómeno activo que no solamente implica explicaciones en clase (método magistral), sino que además los estudiantes aprendan a través del análisis y actividades que realizan, ya que, aprender haciendo, provee un mayor y duradero aprendizaje. Así mismo, este método es muy usado para la formación 
de las competencias directivas y gerenciales de los estudiantes de profesiones relacionadas con la investigación de mercados, la publicidad y la promoción de ventas, la mercadotecnia internacional, la mercadotecnia social, la mercadotecnia internacional y el comportamiento del consumidor.

Siguiendo con el método antes expuesto, García (1997) visualiza el estudio de casos como una técnica que consiste en presentar ante un grupo de alumnos situaciones o problemas (que no son otra cosa que los denominados casos) tomados normalmente de la realidad, para que mediante análisis y discusión por todos los participantes se adopte una decisión. De esta manera, se ambiciona acrecentar en el alumno su capacidad de análisis, decisión y actuación ante los problemas comerciales. Aunque lo anterior es relevante, ya mucho antes se había comprobado la efectividad de los estudios de casos. Frente a esto, García (1974) reconoce que los beneficios del análisis de casos son:

a)Está orientado hacia la situación en la práctica profesional.

b) Prepara la actuación aplicando la metódica del trabajo científico.

c)Amplía la problemática de la solución de las decisiones con las técnicas de procedimiento.

d) Aprovecha el contexto conocido para su transformación metódica con vista a la solución de problemas reales.

e)Educa para una actividad autónoma e independiente en problemas concretos.

f) Forma para trabajar en equipo, con miembros de iguales derechos, en la solución de problemas (p. 18).

Morduchowicz (1995) explica que la educación del marketing utiliza generalmente los canales tradicionales para la enseñanza. Según él, el maestro no puede considerarse como la única fuente de conocimiento y por esto se requiere abrir las puertas del aula para incorporar otra información más allá de la institución educativa, libros o experiencias del profesor. En otras palabras, es necesario incorporar nuevos medios que amplíen las fuentes de información y generen pensamiento crítico en los alumnos. Para ello, se utilizan los productores de contenido, como los hipertextos, imágenes virtuales, blogs, videos, aulas virtuales, wikis, conferencias web, entre otros.

Porlán (1993), en sus investigaciones, promueve la importancia de la inclusión de la reflexión didáctica mediante un proyecto curricular en el que los docentes, que tienen la responsabilidad de crearlo, pongan en relación la práctica con la teoría. Dentro de los elementos que involucra un rediseño curricular, Porlán delimita tres conocidos vértices: la experimentación curricular, el desarrollo profesional y la investigación educativa. Como complemento, González (2006) expresa que 
La función docencia tiene como objeto la circulación de los saberes en la universidad, pero en su desarrollo se manifiesta la función de investigación. Esta, es seleccionado por el currículo con fines pedagógicos, saber enseñar, para formar profesionales que se desempeñarán en las sociedades del conocimiento, el entorno con el cual interactúa la universidad (p. 105).

Así mismo, Angulo, Araúzo y del Olmo (2006) explican que usar metodologías en el proceso de formación basadas en "el cómo" se enseña, frente a "el qué" se enseña, hace que el docente se centre en la propiciación de una experiencia forjando el uso de métodos colaborativos en el aula; además, las consecuencias que se derivan de la misma lo llevan a identificar el "cómo enseñaremos”, es decir, la aplicación de la mejora continua (concepto de corte empresarial). En este sentido ellos proponen un método didáctico centrado en el cooperativismo, el cual se adapta a las necesidades de la enseñanza del marketing en el ámbito de la ingeniería. En sus estudios sobre el uso de esta técnica han logrado los siguientes resultados:

- Los estudiantes, además de descubrir lo que ignoran han aprendido a buscar respuestas, a reflexionar sobre la información obtenida, a trabajar en equipo, a vencer las reticencias, a participar en debates, a exponer sus puntos de vista, a perder la vergüenza, a equivocarse, etc.

- Existe una motivación y satisfacción de los estudiantes frente a sus aprendizajes, consecuencia de la mayor experiencia del profesorado y la extinción del método de clases magistrales.

También cobra importancia el uso de la investigación formativa para la enseñanza del mercadeo. Meroño, Llamas, y Bueno (2013) identificaron algunos resultados de aprendizaje marcados en asignaturas relacionadas con el marketing utilizando la investigación, tales como: los estudiantes logran gestionar la información procedente del entorno para la planificación estratégica de los medios de comunicación y, especialmente, son capaces de buscar con criterio científico información procedente de diferentes contextos y seleccionar dicha información con espíritu crítico. Así mismo, Muro et al., (2017) encontraron que el uso de la investigación en mercadeo permite que los estudiantes consideren que, en general, obtienen el conocimiento teórico necesario para tener un concepto sobre lo que es la creatividad y la innovación, así como lo importante que son estas dos habilidades en el ambiente laboral. Estos dos elementos son considerados de gran importancia dentro de las competencias de un profesional especialista en mercadeo. 
La didáctica es importante para enseñar mercadeo, pues pone en consideración el método para que los estudiantes fomenten sus habilidades y competencias específicas de la materia para un mejor futuro profesional. Pero la didáctica también es importante para el desarrollo de habilidades genéricas como la lectura crítica y la comunicación escrita, transcendentales para el desarrollo de la investigación formativa. Giraldo (2015) establece que a través de la escritura se pretende que el estudiante desarrolle las destrezas comunicativas adecuadas, para un mejor aprovechamiento del quehacer académico-científico. De esta manera, la escritura es un camino para adentrarse en la comprensión disciplinar y evaluar los alcances o límites conceptuales sobre un tema en particular. Por eso, Castronovo y Mancovsky (2010) afirman que los docentes no son ajenos a la tarea de fortalecer la habilidad de la escritura en los estudiantes. Argumentan que "la lectura y la escritura son competencias que necesitan desplegar los estudiantes para el logro de sus aprendizajes académicos y es una tarea propia del docente ayudarlos a que lo logren” (p. 819). Esto también hace parte de la relación intrínseca que existe en la aplicabilidad de la investigación en la enseñanza del mercadeo.

Desde otra perspectiva, Ríos (2006) considera de gran importancia el desarrollo de la investigación en mercadeo desde el enfoque histórico hermenéutico, usando técnicas como la etnografía y la observación no participante. En este caso, los procesos de investigación en la perspectiva crítico social parten de preguntas y de presupuestos teóricos iniciales por parte del investigador. En este sentido, el docente, ha vivido un proceso de inmersión en la comunidad, la conoce porque la ha cohabitado y, en tal proceso, construye las preguntas y las categorías de análisis con la comunidad misma, deriva su saber, devela sus imaginarios, comprende sus particularidades. Así mismo, adquiere conocimiento, desde la praxis, para luego aplicar al proceso de toma de decisiones. Esto también se aplica cuando se quiere analizar las maneras como un consumidor se comporta.

Nuevas investigaciones con herramientas tecnológicas, brindan también apoyo a los diversos esquemas didácticos que usa el docente para la enseñanza del mercadeo. Por ejemplo, Torres y Ferrer (2018) en uno de sus estudios para una asignatura denominada Mercadeo I, establecieron que el entorno virtual de aprendizaje (EVA) - desde el punto de vista estructural y de contenido- contribuye a la formación de los estudiantes a través del dominio de conocimientos, habilidades técnicas, desarrollo de competencias, actitudes y valores propios de las actividades relacionadas con su campo tecnológico que le facilitan la operatividad en el entorno laboral.

Soportando el estudio anterior, Jiménez y Estraño (2017) consideran que "la educación debe ser innovadora, creativa, significativa y vivencial" (p. 19). Por consiguiente, se debe hacer uso de las plataformas tecnológicas según el entor- 
no, para así mejorar el proceso educativo a través de la interacción social. Allí mismo, se generan los procesos de formación y consolidación de nuevos conocimientos y aprendizajes.

\section{Habilidades profesionales en mercadeo}

Ahora se inicia la validación de lo dicho anteriormente, lo cual implica entender cuáles son las habilidades y perfiles profesionales que exigen las empresas de las personas a cargo del mercadeo. Con esto se apoya la tesis de que la aplicabilidad de la investigación formativa y la didáctica promueven el desarrollo de profesionales apasionados por sus áreas y con habilidades diferentes a las genéricas.

Ballesteros, Ponce y González (2016) explican que el elemento que incide en la contratación de un mercadólogo en la PyME se basa en la forma como este persuade al gerente o dueño del negocio en dos aspectos: para orientar las funciones de la empresa hacia el mercado, la consolidación de una filosofía de servicio y en la recolección y administración de la información de los consumidores o clientes de la organización bajo dos principios fundamentales: la innovación y la responsabilidad social.

Así mismo, Víquez (2015) plantea que para el empresario lo que realmente tiene peso e importancia es la actitud, el interés y la pasión mostrada por el candidato hacia el mercadeo. A su vez, demandan personas proactivas, que tengan iniciativa para laborar, que siempre busquen áreas de oportunidad, que su actuar sea bajo el orden, la disciplina, el compromiso y la responsabilidad. Aquí se explica la importancia de la investigación formativa.

Ávila y Muñoz (2017) concluyen que un mercadólogo es el profesionista que trata de anticiparse al futuro. Los conocimientos del mercadólogo tienen que estar basados en las tendencias del mercado que quiere abordar y en las necesidades de los consumidores. El mercadólogo es una persona con la capacidad de análisis, creativo y con toma de decisiones apropiadas. Justamente aseguran que una de las debilidades de los profesionales en mercadeo en su inmersión en el campo laboral, es el desconocimiento del mercado al que quiere aplicar sus estrategias y la falta de la habilidad para la toma de decisiones.

Gracias a los avances en las comunicaciones y a la era de la virtualización de contenidos, las empresas han adoptado un nuevo perfil en las áreas de mercadeo. Así lo refiere Castelló (2010) cuando explica cómo la figura del community manager es el nexo de unión entre la empresa y el público en Internet, quien gestiona la reputación online, posiciona a la organización en el espacio virtual, conversa con la audiencia y crea contenidos para compartirlos. Este tipo de profesional integra actitudes y aptitudes relacionadas con la capacidad de escucha, trans- 
parencia, reflexión, actitud crítica, liderazgo y vocación. Dicho de otra manera, además de la creatividad en el desarrollo de sus funciones también el profesional en mercadeo debe ser capaz de investigar e indagar en otros escenarios diferentes a los que conoce.

A lo anterior, Treviño, Barranquero y Zusberro (2013) muestran el perfil del community manager. Inicialmente, consideran que este tipo de profesional no es estático, sino que está en continua evolución, de ahí que su formación no esté predeterminada. Sin embargo, algunas características que predominan son: la buena redacción, ser un buen comunicador, escuchar y dar respuesta inmediata, la empatía y otras habilidades sociales, así como, tener conocimientos técnicos, actualizarse constantemente y una base de formación en marketing. Todo ello, apunta a lo ya establecido anteriormente cuando se hace referencia a la integralidad en la formación.

Desde otro ángulo, Crissien (2005) asumen que un profesional en mercadeo debe adquirir ciertas habilidades de tipo directivo y gerencial. En este sentido, se parte del hecho de que el ser humano es en esencia un ser de necesidades y el mercadeo se encarga, científicamente, de identificar esas carencias y de desarrollar productos y servicios que las satisfagan y crear relaciones a largo plazo; todo esto se realiza si el profesional tiene habilidades investigativas, así como herramientas personales que forjan su liderazgo, no solamente en su propia vida, sino también en el ámbito laboral. Por ende, se hace necesaria la adquisición de un pensamiento mercadológico o la visión mercadológica del gerente líder.

Y una habilidad, relacionada con el conocimiento mercadológico, que no ha sido valorada del todo es la creatividad, entendida por Conde (2017) como el proceso a través el cual un individuo o un grupo elaboran un producto nuevo y original, adaptándose a las condiciones y finalidades de la situación. La creatividad exigida por las empresas no solamente se enfoca en la generación de ideas y conceptos nuevos, sino también en la gestión para desarrollar investigación y realizar innovaciones que generen valor a las organizaciones y permite desarrollar procesos competentes.

Otra habilidad fundamental que debe ser desarrollada mediante la aplicación de la investigación formativa y la didáctica en los procesos de enseñanza del mercadeo es la inteligencia emocional, que también incide en el desempeño laboral de quienes dirigen o laboran en las organizaciones y empresas. Este término acuñado por Goleman (1995), y el contexto del presente trabajo, se define como la capacidad que tiene el gerente de auto-reflexión, habilidad para reconocer lo que los demás están pensando y sintiendo, conciencia emocional de sí mismo, autocontrol emocional, competencia social, y competencia para la vida y el bienestar. Pero no se desea profundizar tanto en este aspecto, pues sería considerar un nuevo tema de investigación, esto es, la competencia sicosocial y el manejo 
adecuado de las relaciones interpersonales del profesional. Un nuevo discurso con nuevas orientaciones, que para este caso no requieren mayor complejidad de análisis.

Con base en el análisis de las tres categorías establecidas en la metodología de esta investigación, se estableció una red semántica que comprueba la relación entre las mismas, logrando identificar complementariedad para responder la pregunta de investigación ¿Cuál es la importancia de la aplicabilidad de la investigación formativa y la didáctica para la enseñanza efectiva del mercadeo y su debido y adecuado aprendizaje?

Figura 2. Relación entre las categorías de análisis

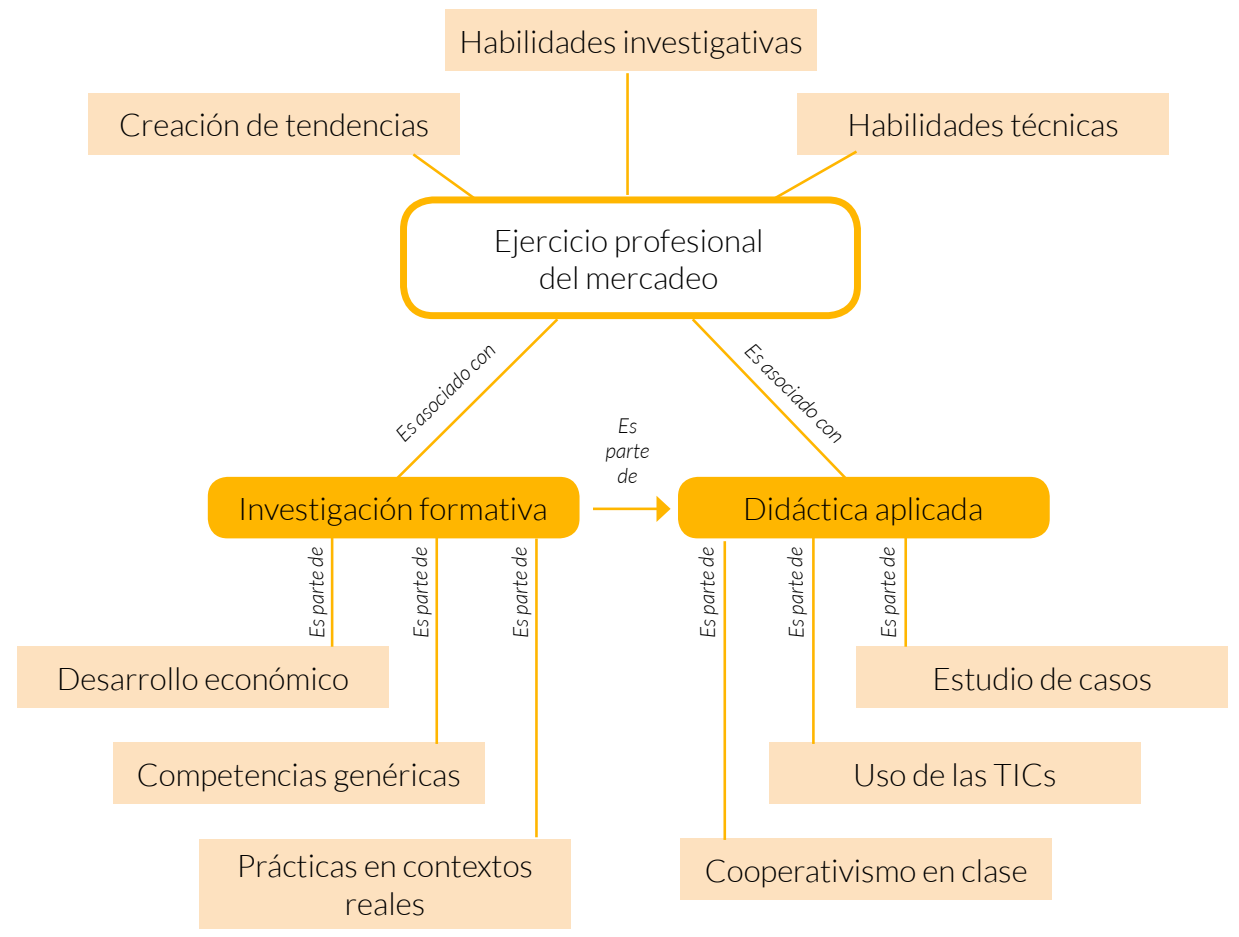

Fuente: elaboración propia

En la Figura 2, primero se establece la investigación formativa como fenómeno indispensable dentro del éxito de las instituciones de educación superior. Su uso, desarrollo e implementación permite que el claustro universitario logre apropiarse de su función de proyección social; además, perfecciona la labor docente, enriqueciendo el fortalecimiento de competencias genéricas (lectura críti- 
ca y comunicación escrita) y específicas (interdisciplinariedad) en los estudiantes a través de prácticas en contextos propios y reales, con las que logran ser partícipes del desarrollo económico para lograr la competitividad de las naciones. En este contexto, Zuluaga (2008) afirma que "la escuela o universidad pierde su norte cuando se preocupa más por la enseñanza que por el aprendizaje [...] y es evaluada más por sus efectos cuantitativos, estadísticos, pero no desde la esencia de su pedagogía” (p. 15).

En segunda medida, la didáctica aplicada a procesos formativos del mercadeo involucra diferentes métodos, en los que se destacan el estudio de casos, el cooperativismo en clase y el uso de las TIC'S para la investigación de escenarios con la actuación especial de los consumidores.

En tercera instancia, la asociación de las dos anteriores categorías conlleva a la formación del ejercicio profesional de aquellos que se dedicarán a la función del mercadeo en las empresas, las cuales exigen personas competentes con habilidades investigativas, y técnicas para la definición de tendencias de mercado. Es decir, la universidad debe lograr, desde la investigación y la didáctica, que los estudiantes de mercadeo tengan un perfil gerencial que se adapte a las exigencias del mismo.

\section{Conclusiones}

Buitrago y Valencia (2008) consideran que el concepto de “empresario" no solamente compete a la disciplina de la administración como un cuerpo de teorías o modelos de comportamiento, como tradicionalmente lo han entendido los científicos sociales, ya que el concepto, las características y el comportamiento del empresario son también modelados por otras disciplinas como la sociología, la psicología y de manera relevante por la economía. Es decir, se requiere una formación integral.

De acuerdo con lo anterior, para lograr esa integralidad en la educación de empresarios, mercadólogos, o como hoy en día son llamados "marketeros", se le debe dar la importancia suficiente a la investigación; y ésta, unida a un proceso didáctico de enseñanza logrará concederles a los futuros profesionales en la rama, las herramientas suficientes para afrontar los retos que le exige el mundo laboral, combinando las técnicas, como el manejo de software y las competencias profesionales como el ejercicio de diseñar e implementar estrategias y comprender mejor el proceso de toma de decisiones.

La aplicabilidad de la investigación formativa y la didáctica para la enseñanza y el aprendizaje del mercadeo, se hace indispensable en nuestros días, aún más cuando hay un cambio de paradigmas enfocados en el ejercicio profesional del 
nuevo egresado de las escuelas o facultades de administración, el cual debe ser preparado para enfrentarse a un mercado cada vez más exigente.

Es importante seguir desarrollando investigaciones en el campo de la formación en mercadeo, con el fin de establecer nuevas relaciones, por ejemplo, entre los estilos de aprendizaje y la investigación de mercados, o en otro sentido, el desarrollo de habilidades técnicas como la debida utilización de las tecnologías de la información siempre en beneficio de una mejor, adecuada y oportuna información para los consumidores. A esto se le une otros conceptos que provienen de la construcción del ser corporativo y del profesional en negocios: la responsabilidad social y la ética empresarial. Temas que se deben vincular a estas nuevas necesidades de investigación.

\section{Referencias}

Agazzi, E. (2001). El desafío de la interdisciplinaridad: dificultades y logros. En Seminario para profesores Departamento de Filosofia Universidad de Navarra. Recuperado de https://dadun.unav.edu/bitstream/10171/5877/1/EVANDRO\%20AGAZZI. pdf [Consultado el 13 de noviembre de 2017].

Angulo, P. S., Benito, de, J. J., Araúzo, A. A. y Olmo, del, R. (2006). Utilización del autoaprendizaje basado en métodos cooperativos para la enseñanza del marketing en carreras de ingeniería. En X Congreso de Ingeniería de Organización, Valencia, 7 y 8 septiembre de 2006.

Ávila, G. V. y Muñoz, A. J. (2017). Estudio sobre el perfil del egresado en un Posgrado de Mercadotecnia en un ambiente de competitividad, en una institución de Educación Superior. Red Internacional de Investigadores en Competitividad, 4(1), 15011521. Recuperado de https://www.riico.net/index.php/riico/article/view/782 [Consultado el 21 de noviembre de 2017].

Ballesteros, S. D., Ponce, E. A. y González, A. J. (2016). Elementos que inciden en la contratación de un mercadólogo en la PyME de Aguascalientes. REVISTA FACCEA, 6(1), 38-46. Recuperado de https://www.udla.edu.co/revistas/index.php/ faccea/rt/captureCite/558/545 [Consultado el 13 de noviembre de 2015].

Buitrago, M. T., y Valencia, J. A. (2008). El empresario en el análisis económico, características y funciones. Ánfora, 15(25), 337-348. Recuperado de https://publicaciones.autonoma.edu.co/index.php/anfora/article/view/193 [Consultado el 16 de enero de 2017]. 
Camacho, M. (2015). El Método del Caso y la enseñanza del marketing. Revista Global de Negocios, 3(2), 61-70. Recuperado de https://papers.ssrn.com/sol3/papers. cfm?abstract_id=2657726 [Consultado el 17 de febrero de 2017].

Carvajal, Y. (2010). Interdisciplinariedad: desafío para la educación superior y la investigación. Luna Azul, 31, 156-169. Recuperado de http://www.scielo.org.co/pdf/ luaz/n31/n31a11.pdf [Consultado el 12 de febrero de 2016].

Castelló, A. (2010). Una nueva figura profesional: el Community Manager. Revista Red Académica Iberoamericana de Comunicación, 1(2), 74-97. Recuperado de https:// dialnet.unirioja.es/servlet/articulo?codigo $=3405401$ [Consultado el 16 de marzo de 2017].

Castronovo, A. y Mancovsky, V. (2010). La lectura y la escritura como procesos centrales de los aprendizajes disciplinares: la inclusión y la pertenencia a una comunidad de lectura específica. En A. Vásquez, M. C. Novo, I. Jakob y Peliiza, L. (Comps.). Lectura, escritura y aprendizaje disciplinar (pp. 819-828). Recuperado de: http:// www.unrc.edu.ar/unrc/comunicacion/editorial/repositorio/978-987-688-007-7. pdf [Consultado el 16 de marzo de 2017].

Civarolo, M. M. (2008). La idea de didáctica. Antecedentes, génesis y mutaciones. Bogotá, Colombia: Editorial Magisterio.

Clark, B. R. (1998). Crecimiento sustantivo y organización innovadora: nuevas categorías para la investigación en educación superior. Perfiles Educativos, 81, 1-16. Recuperado de http://www.redalyc.org/html/132/13208103/ [Consultado el 22 de marzo de 2017].

Conde, R. A. (2017). ¿Cuáles son las principales características de los gerentes modernos que permite identificarlos como líderes en el siglo XXI en las organizaciones? (Tesis de grado, Universidad Militar Nueva Granada). Recuperado de https:// repository.unimilitar.edu.co/bitstream/handle/10654/16695/CondeMartinezRicardoAndres2017.pdf? sequence $=1 \&$ isAllowed $=y$ [Consultado el 25 de marzo de 2016].

Consejo Superior Universitario de la Universidad de Antioquia. (1994). Acuerdo Superior $N^{o} 1$ del 5 de marzo de 1994 por el cual se expide el Estatuto General. Medellín. Recuperado de http://www.udea.edu.co/wps/wcm/connect/udea/582e2ba 1-c2944515-961c-96530772faeb/EstatutoGeneralo7_12_2011.pdf?MOD=AJPERES [Consultado el 16 de octubre de 2017]. 
Crissien, J. O. (2005). Gerencia del Siglo XXI. Revista Escuela de Administración de Negocios, 54, 59-83. Recuperado de https://journal.universidadean.edu.co/index.php/ Revista/article/view/333 [Consultado el 22 de marzo de 2017].

División de Investigación Universidad Nacional de Colombia. (1999). Guía para la Consolidación de un Sistema de Excelencia en Investigación. Espacio Abierto, 4, 9-12. Boletín de la Sede Santafé de Bogotá.

Donoso, G. D. y Vaca, A. V. (2017). La comunicación asertiva en la calidad del desempeño escolar. Recuperado de http://repositorio.ug.edu.ec/bitstream/redug/25408/1/ BFILO-PMP-17P22.pdf [Consultado el 14 de abril de 2016].

García, F. F. (2000). Un modelo didáctico alternativo para transformar la educación: el Modelo de Investigación en la Escuela. Scripta nova. Revista Electrónica de Geografia y Ciencias Sociales, 4(64), 1-24. Recuperado de https://idus.us.es/xmlui/handle/11441/25921 [Consultado el 18 de junio de 2017].

García, R. (1997). La aplicación de las nuevas tecnologías en la enseñanza universitaria del marketing. Revista de Enseñanza Universitaria, 11, 71-81. Recuperado de https://idus.us.es/xmlui/bitstream/handle/11441/11595/file_1.pdf? sequence $=1$ [Consultado el 22 de marzo de 2018].

García, S. (1974). Economía de la empresa y política económica de la empresa. Madrid, España: Esic.

Giraldo, A., Abad, D. y Díaz, E. (2004). Bases para una política de calidad de la Educación Superior en Colombia. Recuperado de https://www.uned.ac.cr/academica/images/ igesca/materiales/o6.pdf [Consultado el 16 de agosto de 2018].

Giraldo, C. (2015). La escritura en el aula como instrumento de aprendizaje. Estudio en universidades. Ánfora, 22(38), 39-58. Recuperado de https://publicaciones.autonoma. edu.co/index.php/anfora/article/view/25 [Consultado el 13 de octubre de 2018].

Goleman, D. (1995). Inteligencia Emocional. Barcelona, España: Kairós.

González, E. M. (2006). La investigación formativa como una posibilidad para articular las funciones universitarias de la investigación, la extensión y la docencia. Revista de Educación y Pedagogía, 18(46), 101-109. Recuperado de https://aprendeenlinea. udea.edu.co/revistas/index.php/revistaeyp/article/viewFile/6938/6351 [Consultado el 22 de abril de 2017]. 
Granés, J. (2001). Principios básicos de la Docencia Universitaria. En: Seminario Internacional Reflexiones sobre Docencia Universitaria. Políticas, Investigación y Evaluación. Bogotá, Colombia: Universidad Nacional Colombia.

Jessup, M. N. y Pulido, R. (1998). Los estudios de calidad de vida: alternativa de educación basada en la investigación. Tecné Episteme y Didaxi TED, 4, 1-14. Recuperado de https://doi.org/10.17227/ted.num4-5698 [Consultado el 8 de noviembre de 2016].

Jiménez, H. J. y Estraño, M. E. (2017). Plataforma Wix como recurso de enseñanza de la asignatura Mercadeo en estudiantes de la Mención Educación para el Trabajo en la FaCE-UC (Tesis de Maestría). Universidad de Carabobo, Venezuela.

Lindsay, R., Breen, R. y Jenkins, A. (2002). Academic research and teaching quality: the views of undergraduate and postgraduate students. Studies in Higher Education, 27, 309-326. Recuperado de https://www.tandfonline.com/doi/ abs/10.1080/03075070220000699 [Consultado el 16 de enero de 2017].

López, G. (2015). La investigación en la formación docente inicial. Una mirada desde la perspectiva socio transformadora. SABER. Revista Multidisciplinaria del Consejo de Investigación de la Universidad de Oriente, 27(1), 143-151. Recuperado de https:/ / www.redalyc.org/articulo.oa?id=427739474018 [Consultado el 22 de marzo de 2018].

Martínez, M. (2004). Los grupos focales de discusión como método de investigación. Heterotopía, 26, 59-72. Recuperado de https://biblat.unam.mx/es/revista/heterotopia/articulo/los-grupos-focales-de-discusion-como-metodo-de-investigacion [Consultado el 22 de noviembre de 2016].

Meroño, C. P., Llamas, G. S., y Bueno, Á. B. (2013). La creación de grupos en Facebook como apoyo a la enseñanza universitaria. Una aplicación en la materia de marketing. Recuperado de https://www.uned.ac.cr/academica/edutec/memoria/ponencias/ parra_sicilia_\%20beltran94.pdf [Consultado el 11 de octubre de 2017].

Moncada, L. I. y Pinilla, A. E. (2006). Investigación en educación. Revista de la Facultad de Medicina, 54(4), 313-329. Recuperado de https://revistas.unal.edu.co/index. $\mathrm{php} / \mathrm{revfacmed} / \mathrm{article} / \mathrm{view} / 43969$ [Consultado el 22 de junio de 2017].

Morduchowicz, R. (1995). La lectura crítica de la prensa en el contexto Iberoamericano. Educación y medios de comunicación en el contexto Iberoamericano. Universidad Internacional de Andalucía. España. 
Moreno, T. (2011). Didáctica de la Educación Superior: nuevos desafíos en el siglo XXI. Perspectiva Educacional, Formación de Profesores, 50(2). 26-54. Recuperado de https://dialnet.unirioja.es/servlet/articulo?codigo $=3681264$ [Consultado el 23 de julio de 2017].

Moreno, J. C. y Ruiz, P. (2010). La educación superior y el desarrollo económico en América Latina. Revista iberoamericana de educación superior, 1(1), 171-188. Recuperado de http://www.scielo.org.mx/scielo.php?script=sci_arttext\&pi$\mathrm{d}=$ S2007-28722010000100013 [Consultado el 23 de julio de 2017].

Muro, S., Christel, J. Calvo, D., Pacheco, M. F., Quintero, D. G. y Reyes, S. I. (2017). Propuesta de un modelo de mercadotecnia holística que permitan profundizar en los conceptos de innovación y creatividad para mejorar las competencias de los egresados de LRC. (Tesis de grado, Instituto Politrécnico Nacional). Recuperado de https://docplayer. es/94094939-Instituto-politecnico-nacional.html [Consultado el 22 de noviembre de 2017].

Neumann, R. (1992). Perception of the teaching-research nexus: A framework for analysis. Higher Education, 23, 159-171. Recuperado de https://link.springer.com/article/10.1007/BFoO 143643

Ortegón, L. F. (2013). Nuevos medios masivos de comunicación y su uso en la enseñanza del marketing (Tesis de Especialización en Docencia Universitaria), Universidad Militar Nueva Granada.

Pérez, E. (2003). Epistemología, currículo y formación docente. Cumaná, Venezuela: Editorial Universitaria

Pina, F. H. (2002). Docencia e investigación en educación superior. Revista de investigación educativa, 20(2), 271-301. Recuperado de http://revistas.um.es/rie/article/ view/98921 [Consultado el 22 de junio de 2017].

Porlán, R. (1993). Constructivismo y escuela. Hacia un modelo de enseñanza-aprendizaje basado en la investigación. Sevilla, España: Díada.

Ries, A., y Trout, J. (1999). La guerra de la mercadotecnia. México, Ed. McGraw.

Ríos, S. C. (2006). La teoría y su función en los tres modelos de investigación. Ánfora, 13(20), 66-76. Recuperado de https://www.redalyc.org/articulo.oa?id=357835619004 [Consultado el 13 de octubre de 2017]. 
Rosselló, M. R. (2005). Didáctica General versus didácticas específicas: un viaje de ida y vuelta. Revista Educació i Cultura, 18, 133-142. Recuperado de https://www.raco. cat/index.php/EducacioCultura/article/view/75935/96550 [Consultado el 15 de octubre de 2017].

Sánchez, M. (2007). Análisis del retorno a la inversión en la cooperación científico tecnológica: Una herramienta para la relación Ciencia-Sociedad en Colombia. Bogotá, Colombia: Cooperación Internacional de Acción Social.

Sánchez, M. Y. (2017). Ambientes de aprendizaje andragógicos. Una perspectiva fenomenológica. Caracas, Venezuela: Universidad Central de Venezuela.

Sandoval, M. (2010). Gestión del conocimiento y competencias: ¿una nueva forma de educar? Ánfora, 17(29), 61-90. Recuperado de https://www.redalyc.org/ html/3578/357835616004/ [Consultado el 20 de marzo de 2017].

Torres, J. y Ferrer, M. F. (2018). Entorno Virtual de Aprendizaje (EVA) para la administración b-learning del curso Tecnología y Práctica de Mercadeo I. Educare, 21(2), 68-78. Recuperado de https://dialnet.unirioja.es/ejemplar/479396 [Consultado el 13 de octubre de 2017].

Treviño, M. P., Barranquero, A. y Zusberro, N. (2013). Community managers: presente y futuro de un perfil profesional emergente en la dimensión 2.0. Su influencia en la rentabilidad reputacional online. Recuperado de https://www.ucm.es/data/cont/media/www/pag-6429/ Trevi\%C3\%B1o_Barranquero.pdf [Consultado el 19 de octubre de 2017].

Villadiego, J., Huffman, D., Guerrero, S. y Cortecero, A. (2017). Base Pedagógica para Generar un Modelo no formal de Educación Ambiental. Revista Luna Azul, 44, 316-333. Recuperado de http://www.scielo.org.co/scielo.php?pid=S1909-24742017000100019\&script=sci_abstract\&tlng $=$ es [Consultado el 13 de octubre de 2017].

Víquez, A. C. (2015). Análisis de las competencias que busca un empleador de la empresa FIFCO en un candidato para un puesto de mercadeo. Recuperado de https://docplayer. es/140497 11-Analisis-de-las-competencias-que-busca-un-empleador-de-la-empresa-fifco-en-un-candidato-para-un-puesto-de-mercadeo.html [Consultado el 22 de junio de 2017].

Zuleta, E. (2010). Educación y democracia: Un campo de combate. Editorial Demófilo.

Zuluaga, R. D. (2008). 300 estrategias de animación a la lectura. Manizales, Colombia: Editorial Manigraf. 